\title{
The effects of quark interactions on the dark matter kinetic decoupling
}

\author{
Kenji Kadota* \\ Department of Physics, Nagoya University, Nagoya 464-8602, Japan \\ E-mail: kadota.kenji@f.mbox.nagoya-u.ac.jp
}

\begin{abstract}
The studies of the dark matter(DM) kinetic decoupling influencing the size of the smallest dark matter halos could provide us the cosmological probe on the nature of the dark matter, in an analogous way that the physics of the baryon decoupling has been unveiling the properties of our Universe through the BAO and CMB. In this talk, the DM kinetic decoupling with the emphasis on DM-quark interactions, in view of the data from the recent LHC and dark matter direct search experiments, will be discussed.
\end{abstract}

VIII International Workshop on the Dark Side of the Universe,

June 10-15, 2012

Rio de Janeiro, Brazil

* Speaker. 


\section{Introduction}

The nature of the weakly interacting massive dark matter still remains an open question despite the growing evidence to support its existence. One of the unique features of the dark matter is that it can, due to its weak interaction and heavy mass, lose the thermal contact with the heat bath at the relatively early stage in the history of the Universe. The decoupling of dark matter from the relativistic plasma has received fair amount of attention, and it led to a compelling interdisciplinary avenue to explore from both particle physics and astrophysics perspectives [1-17].

Even after the dark matter abundance freezes out at the chemical decoupling when the annihilation/creation rates fall well below the Hubble expansion rate, the dark matter still remains in the local thermal equilibrium with the relativistic plasma by the frequent elastic scatterings. At the kinetic decoupling when the temperature drops further, even such scatterings become ineffective compared with the Hubble expansion rate and the dark matter leaves the heat bath staring the freestreaming. The dark matter kinetic decoupling can determine the cutoff scale for the size of the smallest dark matter halo (protohalo) which can have a significant consequence on the structure formation of the Universe.

We pay particular attention to the roles of the DM-quark interactions in the process of kinetic decoupling [1] which have not been fully explored yet compared with the studies on the roles of the DM-lepton scatterings. In addition to the lack of due attention to the DM-quark interactions, another big motivation of our looking into the quark interactions stems from the unprecedented wealth of data from the LHC and direct dark matter search experiments which provide us with the direct probe of the DM-quark interactions. Those particle physics experiments should in principle be able to put the bounds on the dark matter protohalo size useful for the astrophysics studies.

\section{The kinetic decoupling temperature and the mass of the smallest dark matter halo}

Let us first start with the heuristic order of magnitude argument for the kinetic decoupling process. The momentum transfer from the local heat bath with the temperature $T$ to the dark matter $\chi$ per each collision (with an elastic collision rate $\Gamma_{e l}$ ) is small (of order $T$ ) compared with the average momentum of the dark matter around the decoupling epoch, and many collisions $\left(\sim m_{\chi} / T\right)$ would be required for the dark matter of mass $m_{\chi}$ to be in the local thermal equilibrium. The relaxation rate $\gamma$ for the dark matter to establish the thermal equilibrium would hence scale as $\gamma \sim$ $\left(T / m_{\chi}\right) \Gamma_{e l}$ which lets us estimate the kinetic decoupling temperature by requiring the relaxation rate to be of the order of Hubble expansion rate. We refer the readers to Ref. [1] for the more precise estimation using the Fokker-Planck equation. Once given $T_{k d}$, we can estimate the corresponding length scale relevant for the dark matter protohalo mass. The mass of the smallest protohalo is determined by the larger of the DM mass inside the horizon scale at the decoupling and the mass within the free streaming scale.

The comoving free streaming scale $\lambda_{f s}=\int d t(v / a)$ grows logarithmically during the radiation era $\left(a \propto t^{1 / 2}\right)$ and saturates during the matter domination era $\left(a \propto t^{2 / 3}\right)$. The total WIMP mass 


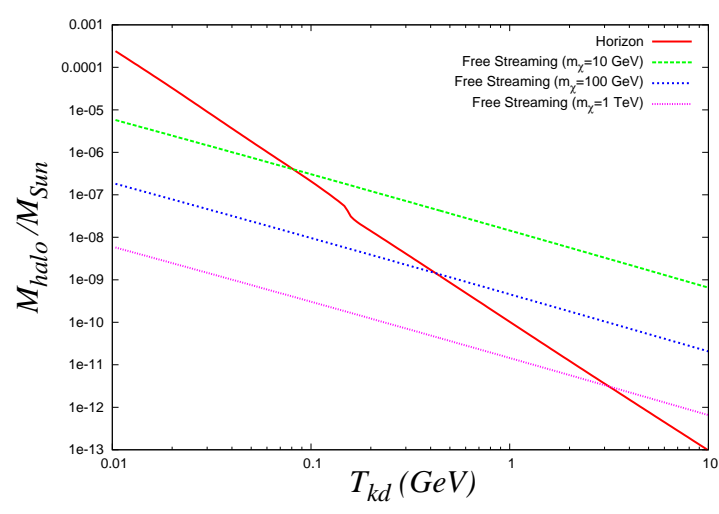

Figure 1: The smallest dark matter halo mass as a function the kinetic decoupling temperature. The smallest mass is the mass within the scale characterized by the larger of the coming horizon size at decoupling and the comoving free streaming length.

contained within the the characteristic scale $k_{*}=2 \pi / \lambda_{f s}$ is

$$
\begin{gathered}
M \approx \frac{4 \pi}{3}\left(\frac{\pi}{k_{*}}\right)^{3} \rho_{m}(a=1) \\
\lambda_{f s}=a_{0} \int_{t_{k d}}^{t_{0}} d t(v / a)=\frac{v_{k d} a_{k d}}{2 a_{e q} C}[\log (C \tau /(2+C \tau))]_{\tau_{k d}}^{\tau_{0}}, C \equiv a_{e q} \sqrt{\pi G \rho_{e q} / 3}
\end{gathered}
$$

where $\tau$ represents the conformal time, $a_{0}=1$ and $a_{e q}$ is the scale factor at the radiation-matter equality. For the dark matter velocity $v_{k d}$, we use $v_{k d}=\sqrt{6 T_{k d} / 5 m_{\chi}}$ with the coefficient $6 / 5$ obtained numerically [11]. This cutoff scale is supplemented by the additional damping due to the acoustic oscillations for the modes whose scales are small enough to couple to the radiation fluids inside the horizon during the decoupling. This cutoff scale is hence characterized by the dark matter mass inside the horizon at the decoupling ${ }^{1}$

$$
M \approx \frac{4 \pi}{3} \frac{\rho_{m}\left(T_{k d}\right)}{H^{3}\left(T_{k d}\right)}=\frac{4 \pi}{3} \rho_{m}\left(T_{0}\right) \frac{h_{e f f}\left(T_{k d}\right)}{h_{e f f}\left(T_{0}\right)}\left(\frac{T_{k d}}{T_{0}}\right)^{3} \frac{1}{H^{3}\left(T_{k d}\right)}
$$

where $T_{0}$ represents the current temperature, $\rho_{m}$ is the DM density and $H\left(T_{k d}\right)$ can be obtained from the Friedmann equation assuming the radiation domination at the kinetic decoupling. $h_{e f f}$ is the effective degrees of freedom for the entropy density and we adopted the model (equation of state B) of Ref. [18] for the effective number of degrees of freedom as a function of the temperature. The horizon and free streaming scales are plotted in Fig. 1. The horizon scale determines the cutoff scale of the smallest halo size for a a wide range of the parameters while the free steaming length becomes more important than the horizon scale for a large $\sqrt{T_{k d} / m_{\chi}}$ as demonstrated in this figure. This behavior is expected because the horizon scale scales as $\tau_{k d} \sim 1 / T_{k d}$ while the free streaming length scales as $\sqrt{T_{k d} / m_{\chi}} \tau_{k d}$.

\footnotetext{
${ }^{1}$ Our expression differs from that of Ref. [11] by a factor $h_{e f f}\left(T_{k d}\right) / h_{e f f}\left(T_{0}\right)$.
} 


\section{Examples}

\subsection{Effective operator approach}

It would be illustrative to consider the effective field theory approach to see the essential features of the DM-quark interactions and their relations to the kinetic decoupling temperature. A great feature of studying such effective DM-quark operators is that we can study the range of the kinetic decoupling temperature allowed by the recent data from the LHC and dark matter direct detection experiments which directly probe DM-quark interactions.

Because our purpose here is to study the potential significance of the DM-quark scattering in the dark matter kinetic decoupling processes which can be constrained from the current LHC and dark matter experiment data, we simply assume the dark matter is a Majorana fermion which is a SM singlet and consider the following effective DM-quark point interaction operators relevant for the direct dark matter search experiments

$$
\begin{aligned}
& \mathscr{O}_{S}=\sum_{q} \frac{m_{q}}{\Lambda^{3}} \bar{\chi} \chi \bar{q} q \\
& \mathscr{O}_{A}=\sum_{q} \frac{1}{\Lambda^{2}}\left(\bar{\chi} \gamma^{\mu} \gamma^{5} \chi\right)\left(\bar{q} \gamma_{\mu} \gamma^{5} q\right)
\end{aligned}
$$

These operators lead respectively to the spin-independent and spin-dependent interactions. The other DM-quark interaction operators besides these scalar and axial-vector operators vanish in the non-relativistic limit for the Majorana fermion dark matter.

We here make a simplifying assumption that the DM universally couples to the Standard Model (SM) up and down type quarks via $\mathscr{O}_{\mathscr{A}}$ while the DM couples through the quark mass suppression factor $m_{q}$ in $\mathscr{O}_{\mathscr{S}}$ inferred from the chirality breaking.

The spin independent dependent cross section per nucleon reads

$$
\sigma_{S I} \approx \frac{4 \mu_{p}^{2}}{\pi \Lambda^{6}} f_{p}^{2}
$$

where $\mu$ is the nucleon-DM reduced mass and we use the default values of the nucleon parameters in DarkSUSY to obtain the effective coupling of the DM with the nucleon $f_{p}$. The spin dependent cross section is

$$
\sigma_{S D} \approx \frac{16 \mu_{p}^{2}}{\pi \Lambda^{4}}\left(\sum_{q} \Delta_{q}^{p}\right)^{2}
$$

where we use the value $\left(\Sigma_{q} \Delta_{q}^{p}\right)^{2} \approx 0.102$ for the spin fraction carried by the quark in the nucleon $[19,20]$. For the dark matter direct search experiment constraints, we used the data from the SIMPLE [21] for the spin dependent cross section and the data from the XENON100 [22] for the spin-independent interaction which are currently most sensitive to the high end of the DM mass compared with the other direct search experiments.

A complimentary set of constraints comes from the collider experiments. The collider constrains have the advantage over those from the direct search experiments which suffer from the astrophysical uncertainties such as the dark matter density and velocity distributions. The recent 


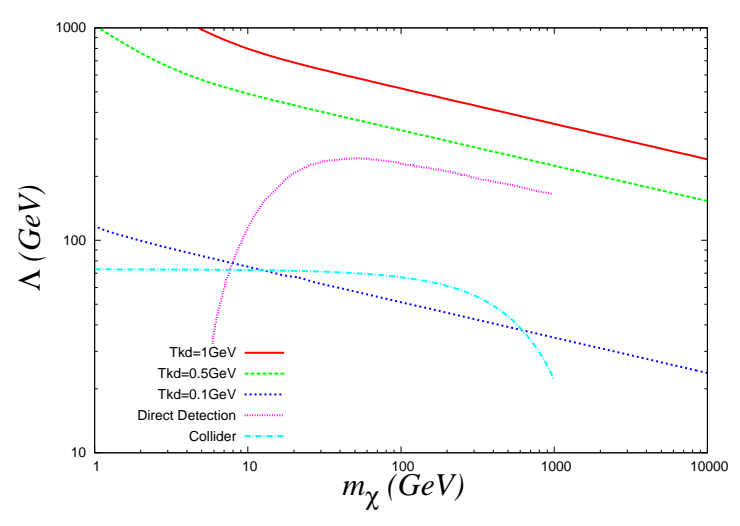

Figure 2: The effective cutoff scale as a function of the dark matter mass for the effective scalar point interaction operator.

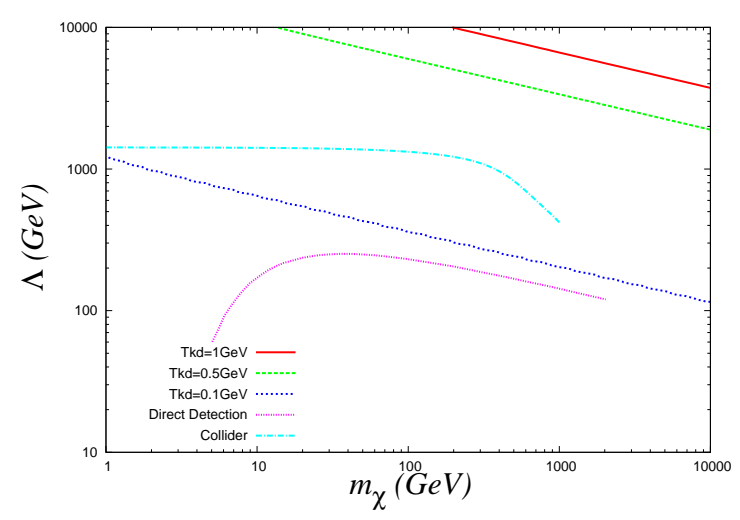

Figure 3: The effective cutoff scale as a function of the dark matter mass for the effective axial vector point interaction operator.

LHC data from the CMS [23] presented the mono jet analysis which required the leading jet with $p_{T}>110 \mathrm{GeV}$, pseudorapidity $|\eta|<2.4$ and the missing transverse energy $H_{t}>350 \mathrm{GeV}$ with 1142 observed events and the Standard Model prediction of $1224 \pm 101$ for the data sample of $4.7 / \mathrm{fb}$ total integrated luminosity at a center-of-mass energy of $7 \mathrm{TeV}$. We consider the collider lower bound of the effective coupling scale $\Lambda$ within $2 \sigma$. We implemented the effective operators by treating the scalar and axial vector operators separately at a time in Madgraph/Madevent. The Pythia was then used for the hadronization including the initial/final state radiations and the jets were treated by the pycell subroutine.

The lower bounds on $\Lambda$ from the direct dark matter search and collider experiments are shown in Figs. 2 and 3 as a function of the dark matter mass along with the contours of the kinetic decoupling temperature. The current lack of the detection of the new physics or dark matter prefers the higher kinetic decoupling temperature. The linear behavior of the kinetic decoupling temperature on the log-scale as indicated in the figures was expected because $T_{k d}$ was obtained from $H\left(T_{k d}\right)=\gamma\left(T_{k d}\right) / 2$ and the the rate coefficient $\gamma$ in the Fokker-Planck equation scales approximately as the powers of $\Lambda, T$ and $m_{\chi}$ in the small momentum transfer limit. 
Our effective-operator analysis provides us with upper bounds on the smallest dark protohalo mass directly from the current LHC and direct dark matter search limits, without having to scan the parameters space of a specific particle model. For instance, we find that for $m_{\chi}=300 \mathrm{GeV}$ the smallest allowed kinetic decoupling temperature is $350 \mathrm{MeV}$ for the scalar operator and 150 $\mathrm{MeV}$ for the axial-vector operator, corresponding to upper limits on the smallest protohalo mass of $3 \times 10^{-9} M_{\odot}$ and $5 \times 10^{-8} M_{\odot}$ respectively. Note that here the protohalo cutoff mass scales as $M_{\text {halo,min }} \propto\left(T_{k d} \sqrt{g_{e f f}\left(T_{k d}\right)}\right)^{-3} h_{e f f}\left(T_{k d}\right)$. Independently of the DM mass and spin-dependent or -independent interaction, there is an absolute lower bounds on the kinetic decoupling temperature of $\sim 100 \mathrm{MeV}$ and a corresponding absolute upper bound of $\sim 10^{-6} M_{\odot}$ (of order of the Earth's mass) on the mass of the smallest dark protohalos.

\subsection{Minimal Supersymmetric Standard Model(MSSM)}

To see the relative significance of the quark interactions compared to the DM interactions with the other particles besides the quarks, we shall present a more model dependent discussion in this section. An advantage of specifying the concrete model is that we can now consider all the the different DM interaction operators including the interference terms which were hard to specify in a model independent approach treating the different effective operators separately.

As a concrete model, we choose the MSSM where $T_{k d}$ turns out to vary in a wide range even beyond $1 \mathrm{GeV}$ for a large parameter space, and hence it would be worth checking the relative importance of the DM-quark interactions compared with the DM scatterings off the leptons which have been discussed extensively in the literature. For the effective number of degrees of freedom, we adopted the model of Ref. [18] and the QCD phase transition temperature was set to 154 $\mathrm{MeV}$ (equation of state B [18], the default model in DarkSUSY [24]). For the scattering of the DM off the quarks, we simply turn on the DM-quark scatterings above $154 \mathrm{MeV}$. Even though the temperature at which the asymptotically free quark description becomes valid is expected to be higher than the QCD phase transition temperature, this simplification would suffice for our purpose of showing the potential significance of the DM-quark scatterings in estimating the dark matter protohalo mass. Choosing a higher temperature for the threshold of the DM-quark scatterings to ensure the free quark descriptions does not change our conclusion of this section that adding the DM-quark interactions in existence of the DM-lepton scatterings can well increase the protohalo mass estimation by a factor 2 or more.

The elastic scatterings off the fermions in the supersymmetric models occur through the exchange of gauge bosons, Higgs and sfermions. We here again use our modified DarkSUSY and we included all the possible dark matter interactions and interference terms in the MSSM for the dark matter scatterings. We scanned the MSSM parameter sets $[1,25]$ and we can see, in Fig. 4, that there exists a wide range of the parameter sets for which the kinetic decoupling can occur above the QCD phase transition scale, as first pointed out in Ref. [9] which performed the analogous numerical scan without including the DM-quark interactions. The open circles represent the $T_{k d}$ including the DM-quark interactions, and the crosses are the cases where we did not include the DM-quark interactions to see the difference. While, for $T_{k d} \lesssim T_{Q C D}$, the inclusion of only leptons as widely done for the treatment of the kinetic decoupling can be justified because the quarks were bounded into the hadrons and those such as pions are much less abundant than the light leptons in the thermal bath, Fig. 4 clearly shows $T_{k d}$ can significantly be influenced by the DM-quark interactions for 


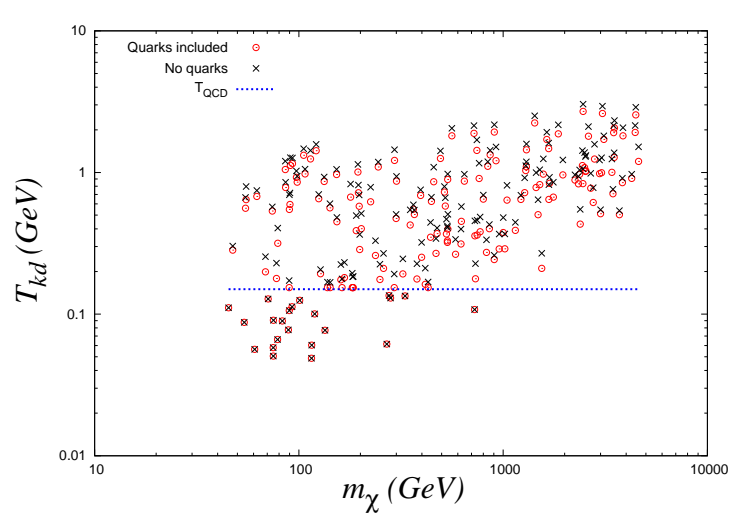

Figure 4: The kinetic decoupling temperature as a function of the dark matter mass where the MSSM parameters were scanned randomly. The inclusion of the DM-quark scatterings can change the kinetic decoupling temperature by $30 \%$ and hence the dark matter protohalo mass by more than $100 \%$.

$T_{k d}$ above the QCD scale where we turned on the DM-quark scatterings. We found the typical ratio of $T_{k d}$ with and without the inclusion of the DM-quark interactions in the MSSM parameter scan ranged between a factor unity and about 1.3. For the dark matter mass range whose protohalo mass is characterized by the horizon size, $M_{\text {halo }} \sim T_{k d}^{-3}$ and hence the inclusion of the quark scatterings can lead to a factor 2 increase in the protohalo mass for a wide range of the MSSM parameter sets. For the dark matter mass range whose characteristic scale is determined by the free streaming length, the mass estimation is affected less because $M_{\text {halo }} \sim T_{k d}^{-3 / 2}$.

\section{Discussion/Conclusion}

The studies of the dark matter can provide us with the unique link between the particle physics and astrophysics. To illustrate such connections to seek for the properties of the dark matter, we paid particular attention to the DM-quark interactions and presented their roles in estimating the dark matter protohalo size in a model independent and a model dependent manner. The constraints on the kinetic decoupling temperature from the current LHC and direct dark matter search experiments turned out to be in the regime of the QCD phase transition, signaling the potential significance of the DM-quark interactions in the DM kinetic decoupling processes. The forthcoming LHC and direct dark matter search data will most likely push this $T_{k d}$ bound up even further, possibly beyond the quark-hadron transition regime so that the simple description of the free quarks can be applicable. More optimistically, if we discover the DM-quark interactions at the LHC or direct detection experiments, it can provide us with the lower bound of the smallest dark matter halo taking account of the additional interactions with the leptons as well.

The creation of the dark matter protohalos and their evolution would certainly deserve the further exploration in view of their potential roles in probing the nature of the dark matter and the structure formation of the Universe. 


\section{Acknowledgments}

This work in part was supported by Grant-in-Aid for the Global COE program and for Scientificc Research from the MEXT of Japan, NSF PHY-0456825 and NASA NNX09AT09AT70G.

\section{References}

[1] P. Gondolo, J. Hisano and K. Kadota, Phys. Rev. D 86, 083523 (2012) [arXiv:1205.1914 [hep-ph]].

[2] C. Schmid, D. J. Schwarz and P. Widerin, Phys. Rev. D 59, 043517 (1999) [astro-ph/9807257].

[3] C. Boehm, P. Fayet and R. Schaeffer, Phys. Lett. B 518, 8 (2001) [astro-ph/0012504].

[4] X. -L. Chen, M. Kamionkowski and X. M. Zhang, Phys. Rev. D 64, 021302 (2001) [astro-ph/0103452].

[5] S. Hofmann, D. J. Schwarz and H. Stoecker, Phys. Rev. D 64, 083507 (2001) [astro-ph/0104173].

[6] V. Berezinsky, V. Dokuchaev and Y. Eroshenko, Phys. Rev. D 68, 103003 (2003) [astro-ph/0301551].

[7] A. M. Green, S. Hofmann and D. J. Schwarz, JCAP 0508, 003 (2005) [astro-ph/0503387].

[8] A. Loeb and M. Zaldarriaga, Phys. Rev. D 71, 103520 (2005) [astro-ph/0504112].

[9] S. Profumo, K. Sigurdson and M. Kamionkowski, Phys. Rev. Lett. 97, 031301 (2006) [astro-ph/0603373].

[10] G. Prezeau, A. Kurylov, M. Kamionkowski and P. Vogel, Phys. Rev. Lett. 91, 231301 (2003) [astro-ph/0309115].

[11] E. Bertschinger, Phys. Rev. D 74, 063509 (2006) [astro-ph/0607319].

[12] J. Kasahara, Ph.D. dissertation, University of Utah (2009; ISBN 9781109295320; UMI microform 3368246); J. Kasahara and P. Gondolo, unpublished.

[13] T. Bringmann and S. Hofmann, JCAP 0407, 016 (2007) [hep-ph/0612238].

[14] T. Bringmann, New J. Phys. 11, 105027 (2009) [arXiv:0903.0189 [astro-ph.CO]].

[15] X. -J. Bi, P. -F. Yin and Q. Yuan, Phys. Rev. D 85, 043526 (2012) [arXiv:1 106.6027 [hep-ph]].

[16] L. G. van den Aarssen, T. Bringmann and Y. CGoedecke, arXiv:1202.5456 [hep-ph].

[17] J. M. Cornell and S. Profumo, arXiv:1203.1100 [hep-ph].

[18] M. Hindmarsh and O. Philipsen, Phys. Rev. D 71, 087302 (2005) [hep-ph/0501232].

[19] J. R. Ellis, K. A. Olive and C. Savage, Phys. Rev. D 77, 065026 (2008) [arXiv:0801.3656 [hep-ph]].

[20] M. Alekseev et al. [COMPASS Collaboration], Phys. Lett. B 660, 458 (2008) [arXiv:0707.4077 [hep-ex]].

[21] M. Felizardo, T. Girard, T. Morlat, A. C. Fernandes, F. Giuliani, A. R. Ramos, J. G. Marques and M. Auguste et al., arXiv:1106.3014 [astro-ph.CO].

[22] E. Aprile et al. [XENON100 Collaboration], Phys. Rev. Lett. 107, 131302 (2011) [arXiv:1104.2549 [astro-ph.CO]].

[23] The CMS collaboration, EXO11059, 4.7/fb (2012)

[24] P. Gondolo, J. Edsjo, P. Ullio, L. Bergstrom, M. Schelke and E. A. Baltz, JCAP 0407, 008 (2004) [astro-ph/0406204].

[25] L. Bergstrom and P. Gondolo, Astropart. Phys. 5, 263 (1996) [hep-ph/9510252]. 